\title{
LA INSTITUCIONALIDAD SOCIOCULTURAL DE LAS COMUNIDADES DE LAGUNA DE PERLAS: HAULOVER Y AWAS, RAAS, NICARAGUA
}

\author{
Brenda Mariela Fletes López ${ }^{[s]}$ \\ Esmirna Raudez Urbina ${ }^{[6]}$ \\ Neidy Gutiérrez Soza ${ }^{[7]}$
}

\section{Resumen}

En las comunidades de Laguna de Perlas; Haulover y Awas, el capital social se fundamenta en sus diferentes estructuras comunitarias. Estas a nivel territorial tienen estructuras tradicionales tales como Consejos de Ancianos, asambleas territoriales y comunitarias, sindico, juez comunal (Wihta).

A lo interno de las comunidades se percibe a las alcaldías como una instancia benefactora; pero, que además necesita articular acciones con los líderes tradicionales, según la Ley de Municipios se le mandata. Asimismo, se reconoce que en los últimos veinticinco años han surgido organizaciones que han apoyado el desarrollo. Organizaciones que en determinado momento han sustituido el papel del Estado, principalmente en el caso de salud y la promoción de la participación comunitaria.

Finalmente, se necesita mejorar la sinergia entre los diferentes actores del municipio, porque según los pobladores, puede ayudar a superar las contradicciones y conflictos entre las diferentes estructuras del poder comunitario.

Palabras claves: Institucionalidad, sinergia, desarrollo con identidad, buenas prácticas.

\section{Introducción}

El municipio de Laguna de Perlas tiene aproximadamente una extensión territorial de 3,876 Km2, conformado por las comunidades: Haulover, Raitipura, Awas, Orinoco, Brown Bank, Marshall Point, Tasbapauni, Kakabila, Set Net, La Fe, San Vicente, Pueblo Nuevo, Pondler, El Pedregal, Chaca- Chaca y El Papel.

Se realza que en el municipio de Laguna de Perlas se han desarrollado varios estudios alrededor de la situación social y económica en general. Entre los cuales reviste

[5] Licenciada en Sociología con Mención en Autonomía. mariellafletes@yahoo.com

[6] Licenciada en Sociología con Mención en Autonomía.

[7] Máster en Antropología Social, Tutora de la Investigación. neidygs@yahoo.com 
de importancia el Informe de Desarrollo Humano de la Costa Caribe, 2005, PNUD. En el mismo, se incluyó un apartado sobre las dinámicas territoriales y biodemográficas de la RAAS, en donde se visibiliza la diversidad cultural existente en los diferentes municipios que constituyen la Región Autónoma Atlántico Sur.

Sin embargo, este potencial no termina de ser visibilizado, ni por la institucionalidad autonómica, ni por quienes promueven el desarrollo local y la gestión ambiental. Los poderes locales autónomos no disponen aún de verdaderos espacios de diálogo con los poderes estatales. Por un lado, están los poderes comunales y, por otro lado, los poderes estatales y los organismos de cooperación, promoviendo el desarrollo local (URACCAN- CEIMM, 2004: 61).

Para alcanzar eventualmente los Objetivos de Desarrollo del Milenio en el 2015 la Sociedad Civil debe trascender su origen urbano y vincularse a las comunidades, comarcas y municipios de la región a fin de dialogar, planificar y formular políticas, programas y proyectos que construyan formas de ciudadanía y gobernabilidad para ser sujetos individuales y colectivos de la sociedad multiétnica e intercultural nicaragüense (Zapata et al.; 2006).

La institucionalidad sociocultural y la solución a través de ella de problemas concretos es un aspecto clave para el desarrollo con identidad. Para que exista plenamente, es necesario fortalecer las capacidades locales de liderazgo, de solución de conflictos y gestión asociativa de los procesos productivos, es decir, su capital social. En ese sentido, el presente proyecto de investigación social cuyo concepto principal se centra en la necesidad de hacer una descripción sobre La institucionalidad sociocultural de las comunidades de Laguna de Perlas, Awas y Haulover.

\section{Revision de Literatura}

El desarrollo con identidad para los pueblos indígenas se asienta en los valores, las aspiraciones y el potencial de los pueblos (Victor Cárdenas, citado por Anne Deruyttre; 2001). Por tanto, el desarrollo y la identidad cultural son dos condiciones que no se excluyen, referencia a un proceso que comprende el fortalecimiento efectivo de los pueblos indígenas, para tener la posibilidad de vivir en armonía con el medio ambiente, en que se genere la pertinente administración de los territorios y recursos naturales en beneficio de una vida de mayor calidad, en que la generación y el ejercicio de autoridad les permita auto determinar su destino, y en el que el respeto a los valores y derechos indígenas, incluyendo derechos culturales, económicos, sociales e institucionales de los pueblos indígenas, de acuerdo a su propia cosmovisión y gobernabilidad se haga realidad (Gutiérrez, N., 2006). 
Rupert Lay: (citado por Duncan Ricardo Masson Cabrera; 2005) plantea que la institución corresponde a "un sistema social, en el que las interacciones que generan ese mismo sistema son reguladas por valores, que aseguran la existencia del sistema y, si es posible, extienden el influjo de esa unidad social". En este orden, Juan Guillermo Villada (2002) ilustra el concepto de institucionalidad, como el soporte a los procesos de desarrollo que supone: a) una actitud y un esfuerzo de concertación entre los actores que actúan en una comunidad, b) el desarrollo de organizaciones de base con capacidad de gestión, y c) la construcción de una relación Estado-Sociedad que favorezca la participación ciudadana y la gobernabilidad.

El concepto de capital social se refiere a las relaciones sociales, los vínculos y la sociabilidad. El concepto se nutre de lo que es el corazón de la sociología: los vínculos entre la persona y la sociedad, la acción social, cómo vivir en común, cómo actuar en forma cooperativa para alcanzar propósitos compartidos, cómo evitar la fragmentación y la atomización (Dagmar, R.; Serrano, C., 2005). Para fines del estudio partimos que la dimensión social de la existencia humana puede ser tan importante como las dimensiones económicas, que lo social subyace a cualquier otra acción económica y en lo político que lo social constituye una dimensión de la calidad de vida tan importante como la economía (Bebbington, A. 2005).

Según Ulloa, L. (2008) la sinergia es un fenómeno que hace que juntos produzcamos más. Para efectos de este estudio entenderemos por sinergia la capacidad y disponibilidad de los diferentes actores sociales para articular esfuerzos de intervención a distintos niveles en las instituciones regionales, municipales y comunitarias para alcanzar un desarrollo con identidad

\section{Materiales y Métodos}

El presente trabajo de investigación es de carácter social-etnográfico con enfoque cualitativo. De igual manera, en el presente trabajo se hizo una descripción o reconstrucción analítica de los diferentes escenarios socioculturales que forman parte de la vida en las comunidades.

La investigación se desarrolló en las comunidades de Laguna de Perlas, Haulover y Awas. La composición étnica de estas comunidades se divide en miskitos, creoles y mestizos. Para el desarrollo del estudio se realizaron diferentes procesos en los que se vinculó a las diferentes instituciones socioculturales (líderes, liderezas, autoridades comunitarias, la familia) de las comunidades de estudio.

Se realizaron tres visitas a las comunidades en coordinación con las autoridades locales y comunitarias para presentar el proyecto de investigación y particularmente para un acercamiento y familiarización con la comunidad. El procesamiento de la 
información se realizó en los programas de Microsoft Word y Excel. Luego se hizo una presentación preliminar de los resultados en la comunidad con la finalidad de compartir y recibir retroalimentación por parte de las comunidades. Finalmente se procedió a la elaboración del informe final.

\section{Resultados y Discusión}

\section{Aspectos biodemográficos de las comunidades de Laguna de Perlas: Halouver y Awas}

En relación a los aspectos biodemográficos se ha realizado una síntesis de todos aquellos aspectos, principalmente en aquellos asociados con la población, etnia, migración y la situación de las mismas comunidades en cuanto a salud, educación, vivienda y estrategias de vida.

En relación a la población, según los Planes de Fortalecimiento Institucional Municipal de Laguna de Perlas (PFIM) 2008, plantea que Awas tiene una población aproximada de 100 personas, Halouver de 1,400 habitantes y el casco urbano de Laguna de Perlas de 4,782.

En las tres comunidades cohabitan miskitos, mestizos y creoles, en el caso de Laguna de Perlas la población es mayoritariamente creol. En el caso de Awas y la Halouver población es mayoritariamente miskita. Los mestizos representan una minoría en estas comunidades. Estas comunidades también enfrentan la compleja situación relacionada a la legalidad de sus territorios, la usurpación de sus tierras producto de la migración de mestizos en los últimos treinta años aproximadamente. Naturalmente estos procesos migratorios son la causa principal de la recomposición étnica en estas comunidades.

Por otro lado la migración de jóvenes de las comunidades, producto de la crisis económica que viven en sus comunidades se han visto obligados a migrar fuera de sus comunidades en busca de empleo. Las familias, ante la ausencia de políticas públicas que respondan a sus demandas y necesidades básicas apoyan a sus hijos jóvenes a marcharse de sus comunidades a buscar empleo, es decir que la migración laboral es la mejor alternativa para el aseguramiento de ingresos a los hogares. No se cuenta con datos en cifras que reflejen los ingresos que a través de las remesas ingresan a la economía comunitaria y por ende de la región.

En relación a los servicios de salud y educación, en las comunidades de Lagunas de perlas, Halouver y Awas se cuentan con primaria y secundaria, en donde Laguna de Perlas dispone con mayores y mejores condiciones de infraestructura para salud y educación. En cambio, en las comunidades de Laguna de Perlas y Halouver cuentan 
con servicio de salud (centro de salud y personal médico), y en el caso de Awas las familias son atendidas en el centro de salud de Laguna de Perlas.

El crecimiento desordenado de estas comunidades agudiza la capacidad de cobertura en salud y educación principalmente de parte del Estado. Esta situación genera conflictos en las comunidades, porque las demandas de servicios rebasa la capacidad de atención y cobertura.

Laguna de Perlas cuenta con 420 casas de habitación de las cuales según información del Plan de Fortalecimiento Institucional (PFIM 2008), 70 casa están en mal estado y 100 en regular estado, se puede suponer que en Laguna de Perlas 170 casas no cumplen las condiciones mínimas para ser habitadas.

En el caso de Halouver cuentan con 150 casas de las cuales 20 están en mal estado y 40 en regular estado, estos datos nos indican que 60 casas no reúnen las condiciones mínimas para ser habitadas. En Awas hay 2 casas en mal estado y 10 en regular estado, es decir, que de las 18 casas que existen en total en la comunidad, solamente 6 casas tienen las condiciones mínimas para ser habitadas.

La situación económica de la Región Autónoma Atlántico Sur y en particular del municipio de Laguna de Perlas se caracteriza por la pobreza reflejada en comunidades como ejemplo Awas, en donde se carece de los servicios básicos para satisfacer sus necesidades y tener una vida saludable.

La principal actividad económica de esta comunidad es la pesca, la cual se realiza en Laguna de Perlas y en los ríos cercanos a las comunidades. En su mayoría las familias pescan para el auto consumo. En menor cantidad la pesca se comercializa en la comunidad principal del municipio en Laguna de Perla y en los centros de acopio que por lo general son propiedad privada de personas ajenas a las comunidades y en la mayoría de los casos estos imponen el precio de los productos.

Asimismo, la agricultura es una de las actividades productivas que también realizan en las comunidades. Básicamente producen granos básicos, musáceas, raíces y tubérculos, para el autoconsumo y muy poco para comercio local. Otra forma de ingreso a la economía familiar es a través de las remesas procedente principalmente de los Estados Unidos y Costa Rica; estos son invertidos en algunos casos, en pequeños negocios como pulperías, venta de pan, pesca y ropa.

En las comunidades también se cuenta con profesionales y técnicos que trabajan como servidores públicos en la alcaldía, en el sistema de salud, educación, Poder Judicial, Policía Nacional, entre otras instituciones del Estado con presencia en la comunidad. También trabajan como promotores y oficinistas en Organismos No Gubernamentales como FADCANIC y Acción Medica Cristiana particularmente. 
En las comunidades cuentan con cooperativas de pescadores, dirigentes comunales, brigadistas de salud, brigadas ecológicas, jóvenes (mujeres que cursan estudios universitarios (profesionalización), en las universidades de BICU y URACCAN. También las comunidades cuentan con representaciones en la Asamblea Comunitaria, Consejo Regional y Consejo Municipal entre otras instancias.

\section{La institucionalidad sociocultural de las comunidades}

Las comunidades autodefinen su institucionalidad siguiendo sus propias reglas, principios y valores que han regido históricamente el comportamiento de las personas que están a la cabeza del desarrollo de sus comunidades. Basado en lo anterior, el abordaje de la institucionalidad sociocultural de las comunidades de estudio se inicia a partir de la descripción del funcionamiento del sistema social de organización de las comunidades de estudio. Por tanto, en este particular, en las comunidades de Laguna de Perlas, Awas y Halouver la organización comunitaria se pueda clasificar en territorial, comunitario, municipal y regional. A esto también, tenemos que agregarle las instituciones del Estado y las ONG, con presencia en dichas comunidades.

A nivel comunitario y territorial en las comunidades se cuentan con consejos de ancianos, asambleas territoriales y comunitarias, síndico, juez comunal (Whita). Estas estructuras organizativas cuyo origen es ancestral, han asumido diferentes roles en las comunidades en el marco de la toma de decisiones colectivas para el beneficio comunitario. Entre estos roles, podemos mencionar que las asambleas comunitarias y territoriales han sido el espacio para la toma de decisiones en donde participa toda la comunidad. Esta última (asambleas territoriales) se han conformado a raíz de la aprobación de la Ley de Demarcación Territorial y por ende sus funciones están vinculadas a la administración del territorio.

El síndico es la persona encargada de velar por el uso adecuado de los recursos naturales, mientras que el juez comunal se encarga de ejercer justicia de acuerdo a los valores y normas de la comunidad.

En los años 90 `s en las comunidades surgen las estructuras municipales, llevando a conformar las autoridades municipales. En este espacio de organización comunitaria está conformado por el alcalde, vice alcalde, consejo municipal y sus organizaciones comunales (CDM, CPC, etc.). El gobierno municipal cuenta con una institucionalidad que se expresa a través de la Ley de Municipio y en tanto se establecen normas, instrumentos y reglas que se deben de cumplir relacionado con el desarrollo de las comunidades que forman parte del municipio.

A pesar que en la Ley de Municipio en el Artículo 67 se estable que la alcaldía reconoce la estructura organizativa de las comunidades indígenas... "Asimismo, respetará a sus autoridades formales y tradicionales, a quienes deberán de tomar en cuenta en 
los planes y programas de desarrollo municipal y en las decisiones que afecten directa o indirectamente a su población y territorio".

A lo interno de las comunidades se percibe a las alcaldías como gobierno benefactor, es decir, que es la instancia que tiene que resolver los problemas del municipio y por ende satisfacer las necesidades básicas del municipio. Y al no cumplir con lo anterior también se le ve como el gobierno que no hace nada por el municipio. Este modelo o forma de gobierno (municipal), la población lo vincula directamente con partidos políticos, no como una expresión libre de la sociedad como tal, sino que todo responde a un criterio de gobierno y/o partido político.

De igual manera, se considera que esta institucionalidad propia de las comunidades se ve obstaculizada y en cierta medida acaparada por un nuevo concepto de gobierno; alcaldía municipal (gobierno municipal). Lo anterior, debido a que en la memoria colectiva, de creoles y miskitos, de las comunidades de Laguna de Perla, Awas y Halouver, recuerdan y añoran aquellos tiempos en donde sus comunidades se organizaban para el bien común. Cuando se tenía el control y gestión de los recursos naturales, y principalmente del territorio. La forma de organización en aquel entonces se centraba fundamentalmente en el Consejo de Anciano formado por ancianos líderes y sabios. El Síndico representa los intereses alrededor de los recursos naturales y tiene en su poder los títulos reales de la comunidad.

También existía la autoridad máxima; la Asamblea Comunal en donde se tomaban las decisiones más importantes sobre recursos naturales, territorio y elección de lideres principalmente. Según los ancianos y líderes en la actualidad el liderazgo comunitario esta en crisis y las comunidades en general están conscientes de la gravedad de la situación. Se esta sustituyendo la visión de colaboración y solidaridad por una visión de negocio y competencia. Esto en consecuencia ejerce presión sobre los pocos recursos que aun existen en las comunidades.

..."Para elegir un líder se hacia a mano alzada y sin temor se expresaba el respaldo a la persona (...) ahora las cosas no se hacen así; el voto es secreto y con temor"...

Comunitario de Laguna de Perlas, 2009

...una vez (tiempo de Somoza), vino un hombre importante a Laguna de Perlas, reclamando tierras, pero necesitaba que el sindico respaldara frente a la Asamblea Comunal que esas tierras eran de él; entonces entregó al sindico 250.00 (doscientos cincuenta córdobas), el sindico tomo el dinero (...) después en la asamblea saco el dinero y le dijo al hombre: "Estas tierras que reclamas no son tuyas, porque de lo 
contrario no me hubieras dado este dinero, y devolvió el dinero al hombre frente a toda la comunidad...

Mujer Comunitaria de Laguna de Perlas, 2009.

Este es uno de los valores basado en principios del liderazgo ancestral que en la actualidad los ancianos y ancianas recuerdan con mucha nostalgia y con una luz esperanzadora en sus ojos; como señal que aún existe la posibilidad de recuperar ese liderazgo, que anteponían lo intereses colectivos; el amor al territorio y sus recursos naturales por encima de los intereses individuales.

También se reconoce, aunque no justifican, el actuar de algunos líderes que por la situación de pobreza y motivados por los intereses de los partidos políticos nacionales pudientes, han actuado en contra de los intereses de la comunidad. Sienten que la presencia de los partidos políticos en las comunidades ha contaminado el liderazgo histórico. Pero también, se menciona y reconoce de forma categórica que existen buenas ideas e intensiones por parte de los gobernantes y la legislación nacional, como la existencia misma de todo un cuerpo de leyes (Ley No. 28; Ley de Lengua; Ley de Municipio; etc.) que reconocen el concepto de territorio y propiedad comunal. Al igual que los deberes y derechos que como pueblos indígenas y comunidades étnicas se tienen. Sin embargo, hace falta dar seguimiento al cumplimiento de estas leyes. Para un ejercicio real de autonomía comunitaria y regional. En ese sentido se puede reflexionar sobre la necesidad de que el Estado nacional, gobierno regional y municipal se pongan de acuerdo y desarrollen políticas institucionales que respalden el entusiasmo de las comunidades.

"La Ley 445 es una de las cosas más bonitas que nos pudo ocurrir en estos tiempos"

Comunitaria de Halouver, 2009.

Varios comunitarios y comunitarias coinciden que la Ley No. 445 es el instrumento legal que respalda la autonomía comunitaria. Aquí se establece a "la Asamblea Comunal como la máxima autoridad de las comunidades indígenas y étnicas”.

En esta misma ley se establece que los tres niveles de gobiernos: nivel municipal, regional y nacional deben de respetar y reconocer la propiedad comunal de las tierras y apoyar técnicamente a las comunidades para el proceso de aprobación y aprovechamiento racional de sus recursos.

La Ley No. 445 es una ley que da apertura a las comunidades de participar en un 100 por ciento en todo lo que tiene que ver con la comunidad (...) demarcación territorial, los recursos naturales (...) pero veo también que el gobierno comunal tiene un contrincante; el gobierno municipal 
Esta situación ha debilitado la institucionalidad comunitaria, la participación de las comunidades en las asambleas ha ido disminuyendo. Históricamente a las asambleas comunitarias que se convocaban, asistían al menos un miembro en representación de cada familia. Esto era ley o una norma que en la actualidad se ha ido perdiendo. Hay desconfianza según los comunitarios en los resultados, pues siempre son los mismo, porque en las comunidades se sigue igual.

También comentan los ancianos de las comunidades que "antes", cuando los líderes tenían que salir a resolver asuntos relacionados con conflictos por tomas de tierras o conflictos relacionado con madera: la comunidad se reunía y entre todos hacían una colecta, cada quien ponía lo que estaba en sus posibilidades (gallinas, huevos, yuca, frutas, etc.), estos productos se vendían en Bluefields y con el dinero uno o dos representantes viajaban a Managua para resolver el problema.

"Ahora los lideres sólo quieren resolver los problemas de la comunidad en Managua (...) muchos de ellos sólo piensan en su propio beneficio".

Comunitario de Laguna de Perlas, 2009

En la Ley No 445 el artículo 34 establece: "los tributos recaudados por el fisco en concepto de aprovechamiento de recursos naturales en las Regiones Autónomas deben de beneficiar directamente a las comunidades indígenas en cuyas áreas se encuentran los recursos naturales. La distribución será así: a) un 25\% para la comunidad o comunidades indígenas en donde se encuentre los recursos a aprovechar; b) un $25 \%$ para el Municipio en donde se encuentra la comunidad indígena; c) un 25\% para el Consejo Regional correspondiente; y e) un 25\% para el Gobierno Central. Este 25\% que por ley corresponde a las comunidades es el que se "utiliza" en la actualidad para resolver los problemas de la comunidad. Pero la decisión de hacer uso de ese dinero no es compartida en las comunidades, en la mayoría de los casos una sola persona toma la decisión.

Esto hace que hoy en día la solidaridad no sea una práctica para resolver los problemas de las comunidades relacionados a la usurpación de tierras, uso inadecuado del recurso bosque y el recurso pesca. Al igual que los problemas de droga y seguridad comunitaria. La vida en las comunidades de acuerdo a sus costumbres, valores en donde se colocaba a la familia en una posición privilegiada, es decir era el espacio fundamentales para la trasmisión de conocimientos, saberes, valores y fortalecer la identidad comunitaria.

Ahora se refleja en las conversaciones de los padres de familias, de los abuelos y abuelas, principalmente su preocupación sobre los cambios que han sufrido en lo referente a la vida en familia en sus comunidades. 
...antes por las noches se reunía papá, mamá y alrededor los hijos (...) acompañados de una buena pana de pijibay y elotes cocidos y se hablaba de la comunidad... (Comunitario de Laguna de Perlas).

Las comunidades disponen de energía eléctrica (Laguna de Perla y Halouver), y varias casas tienen televisión y en las mismas comunidades consideran que los programas de televisión ocupan gran parte del tiempo que antes era para trasmitir la historia de la familia, de la comunidad y sus ancestros.

La educación basada en los ejemplos y consejos forma parte de la institucionalidad histórica de las comunidades. Asimismo, el espíritu al trabajo colectivo, el valor de la palabra, el amor al territorio, la cosmovisión, la cooperación, la solidaridad, práctica de medicina natural, formas de elección de líderes y liderezas, el respeto y la espiritualidad.

También se reconoce que en los últimos veinticinco años en las comunidades han surgido organizaciones que en diferentes circunstancias han trabajado apoyando el desarrollo de las mismas comunidades. Estas organizaciones en determinado momento han sustituido el trabajo del Estado, principalmente en el caso de salud y a la promoción de la participación comunitaria. Organizaciones como: FADCANIC, AMC, CEDEHCA y las universidades BICU y URACCAN han servido como facilitadores de procesos encaminados a fortalecer el liderazgo y organización comunitaria, como estrategias en la lucha contra la pobreza.

Entre los procesos que se mencionan: acompañamiento para la consulta de la Ley No. 445, el Sistema Educativo Autònomo Regional (SEAR), Modelo de Salud, Reglamento Interno de las Autoridades Territoriales, Salud Comunitaria, Agua y Saneamiento, Becas para estudios universitarios, Planes de Desarrollo Comunitarios, Radio Emisora Comunitaria, Técnico Superior en Pesca, Salud Sexual Reproductiva, Derechos Humanos, Derechos de la Niñez y la Mujer, Crédito, Capacitaciones y Mediación y Negociación de Conflictos, entre otros componentes de trabajos.

Por lo antes expuesto estas organizaciones son consideradas parte de la institucionalidad sociocultural de las comunidades. Es importante mencionar que los funcionarios de estas organizaciones que trabajan en las comunidades, son profesionales originarios de Laguna de Perlas. Posiblemente sea este uno de los elementos que hace que en las comunidades sientan a estas organizaciones como parte de la comunidad.

De igual manera, además de las instituciones comunitarias, es válido mencionar que en la comunidad también existen Organismos no gubernamentales (ONG`s) e instituciones de asistencia social quienes en gran medida han contribuido a fortalecer el capital comunitario. Entre estas podemos mencionar a Acción Medica Cristiana, 
FADCANIC, MAGFOR, DANIDA, WWF, CEDEHCA, MINED, MINSA, Policía Nacional, Ejército Nacional, URACCAN.

\section{Buenas prácticas: desarrollo con Identidad}

Las "Buenas Prácticas" entendidas como acciones que orientan los comportamientos hacia la creación de valor, focalización en las personas, innovación y generación de resultados medibles y demostrables frente la comunidad y las autoridades.

Max Weber plantea que la sociedad está configurada por los hechos sociales, los cuales son el resultado acumulado de las acciones individuales. Por ello en este apartado se refiere a las acciones que han desarrollado (organizaciones e instituciones) y que las mismas a criterio de las comunidades son consideradas como indicadores de "Buenas Prácticas", porque han contribuido al desarrollo.

En las comunidades se reconoce como una buena práctica; el haber logrado la producción de sus propias normas (Reglamento interno de las Autoridades Territoriales de las Diez Comunidades Indígenas y Afrodescendientes de la Cuenca de Laguna de Pearl Lagoon). Se refieren a este proceso como un aporte orientado a la construcción de su propio desarrollo. Proceso facilitado por la URACCAN en el 2007 y que contó con el apoyo financiero de la cooperación internacional a través de la Fundación Ford.

URACCAN ha acompañado procesos para el fortalecimiento del capital comunitario, a través de capacitaciones a sus líderes (...) acompañamiento en el proceso de demarcación territorial y resolución de conflictos.

Mujer profesional originaria de Laguna de Perlas, 2009

El Reglamento interno se suma a los instrumentos jurídicos que respaldan y defienden los derechos de las comunidades afrodescendientes sobre su territorio. Es decir, que se convierte en una herramienta más, con la cual cuentan para defender sus derechos territoriales y de organización.

La Fundación para la Autonomía y Desarrollo de la Costa Atlántica de Nicaragua, (FADCANIC) existe desde 1990. El trabajo desarrollado por FADCANIC, en donde se ha acompañado a las comunidades al uso productivo y sostenible de recursos; valorizando sus propios saberes y prácticas tradicionales sobre cómo manejar y cuidar los recursos que forman parte de sus vidas e historia. Lo anterior se afirma en la voz de un líder comunitario de Haulover, quien dice:

"FADCANIC es una de las organizaciones que siempre ha estado con nosotros en las buenas y en las malas". 
Para la ciudadanía de las comunidades la metodología de trabajo de FADCANIC ha contribuido al fortalecimiento de su identidad y a mejorar sus estilos de vida. A manera de ejemplo mencionan el Centro Wawashang; cuya misión es desarrollar capacidades en el manejo sostenible de los recursos naturales para que de esta manera logren mejorar la calidad de vida con énfasis en el desarrollo humano.

"FADCANIC siempre ha trabajado de la mano de los hombres y mujeres de las comunidades"

Mujer de Awas, 2009.

FADCANIC ha venido trabajando con las diferentes comunidades y pueblos indígenas a partir de las demandas, potencialidades y oportunidades desde las propias comunidades. Principalmente han desarrollado un trabajo integral, fortaleciendo las capacidades locales y liderazgo natural. Esto apoyándose en las organizaciones locales y tradicionales de las comunidades para el desarrollo de su trabajo.

De igual manera, se reconoce a AMC como una de las pocas organizaciones que desde su llegada al municipio ha trabajado con las comunidades a partir de sus propias potencialidades recursos y capacidades. Contribuyendo de esta manera a mejorar las condiciones de vida de las familias dentro de su propio contexto sociocultural.

FADCANIC y AMC han logrado sistematizar sus experiencias y lecciones aprendidas de cada uno de los procesos que han desarrollado. El documentar y poner a la disponibilidad de las mismas comunidades la información y conocimiento construido, ha permitido un intercambio con los diferentes actores interesados en promover procesos de desarrollo comunitario.

De igual manera, por las acciones desarrolladas por las universidades, FADCANIC y AMC son consideradas como buenas prácticas comunitarias. Es decir que son acciones que forman parte de la historia de la comunidad y que las mismas deben de profundizarse y ser trasmitidas de generación en generación.

\section{Capital comunitario: fortaleza, confianza y oportunidad}

La persistencia histórica de las comunidades, como unidad geográfica de agrupamiento socio-étnico, constituye uno de los principales activos del capital social comunitario de la Costa Caribe (PNUD, 2005). El mismo informe referencia la trayectoria que evidencia que la "comunidad/territorio", no sólo es un espacio con cualidades físicas, climáticas, ambientales, sino un "espacio socialmente construido", base de la identidad, del desarrollo y acumulación del capital cultural y social.

Para entender el significado del capital comunitario se debe partir de la dimensión social de la existencia humana puede ser tan importante como la dimensión 
económica, que lo social subyace a cualquier otra acción económica política (es decir, que todo está integrado); y que lo social constituye una dimensión de la calidad de vida tan importante como la economía.

Varios estudios realizados en las comunidades de Laguna de Perlas, Awas y Halouver coinciden en que el potencial social comunitario se reconoce en las comunidades a partir de su estructura "organizativa y de gobierno", en donde también se identifican a diferentes actores: como las instituciones del Estado, ONGs nacionales e internacionales, concejales regionales, concejales municipales, proyectos, las iglesias, las universidades, y las organizaciones comunales (consejo de anciano, asamblea comunal, la familia entre otras).

Esto nos ilustra el potencial que significa el capital social comunitario para facilitar procesos de planificación y gestión que contribuya a un desarrollo con identidad de las comunidades. Es importante tener presente que por historia las comunidades se caracterizan por compartir en los diferentes escenarios de sus vidas desde lo económico, social, cultural y religioso.

También en el IDH se hace referencia al sistema institucionalizado de cooperación comunitaria. Como un sistema complejo, que han venido construyendo a lo largo de su historia los indígenas y afrodescedientes, a partir de sus propias cosmovisiones, sustentado en la noción de bien común, en un cuerpo de principios y valores 囚donde la unidad y reciprocidad ocupan un lugar central冈, que orientan y alimentan todo el sistema, de normas, sanciones, de estructuras de gestión y gobierno

En el mismo Informe de Desarrollo Humano de la Costa Caribe se hace hincapié en la existencia de este capital, el cual tiene una importancia estratégica para la gobernanza de la reproducción económica, social, cultural de los pueblos indígenas y comunidades étnicasy, la construcción misma de la institucionalidad de la autonomía, que no termina de reconocer a la organización comunitaria, como la base del poder local autonómico.

A partir de lo anterior algunos líderes y maestros de las comunidades consideran que urge que se inicie un proceso de modificación de políticas y estrategias nacionales e internacionales dirigidas a las comunidad indígenas y afro-descendientes de la RAAS y especialmente a las del municipio de Laguna de Perlas, y que se les permita a las comunidades diseñar su propio desarrollo.

A través del reconocimiento y auto reconocimiento de sus capacidades existentes en las comunidades para asumir desde sus propias creencias, costumbres y cultura su propio desarrollo. Potenciando y aprovechando aquellos recursos tangibles e intangibles como sus conocimientos ancestrales, historia y tradiciones, sistema de valores, 
creencias, formas de organización tierra, territorio y recursos naturales que forman parte de su identidad.

"La Institucionalidad sociocultural de las comunidades: gobierno comunitario, instituciones, las iglesias y las familias"

Mujer profesional de Laguna de Perlas, 2009

La familia en las comunidades constituye el principal grupo de pertenencia y referencia del ser humano por ser su grupo original de integración social (Nuevo FISE; 2009). El trabajo en las comunidades debe desarrollarse de forma armónica como factor de confianza y colaboración entre lo familiar, la comunidad y las instituciones; para ello se identifican tres dimensiones fundamentales de las cuales se desprendan acciones operativas que surten efectos directos en las familias de las comunidades.

Es decir que se procure la construcción colectiva de conocimientos, en donde se incluyan la visión de desarrollo de todos los actores, principalmente el de las familias y por ende el de las propias comunidades. Revitalizar los conocimientos ancestrales para la administración y gestión de los recursos y territorio es la preocupación de los ancianos y ancianas de las comunidades.

Sin embargo, también les preocupa que los jóvenes en la actualidad estén contaminados con una visión de negocio, de competencia y no de colaboración y confianza en las capacidades locales para superar la crisis y proteger el territorio y la madre naturaleza.

La lógica que hacia funcionar a la comunidad anteriormente, es la de la supervivencia, convivencia con los recursos naturales (...) no tener una visión más allá de asegurar la alimentación, la educación y la salud de sus hijos (..) ahora esto ha sido cambiado por una visión de acumulación de riqueza.

Profesional de Laguna de Perlas, 2009.

Al valorar lo anterior, se puede afirmar que se debe de apuntar a consolidar el capital comunitario, así mismo fortalecer el liderazgo natural y la identidad. Entendiéndose que el fortalecimiento de la identidad no debe de ser en base de que uno es mejor que otro, sino que en la diversidad étnica y cultural esta la fortaleza y oportunidad para alcanzar el desarrollo.

Funcionarios de organizaciones externas, profesionales originarios de Laguna de Perlas y líderes de las comunidades coinciden que en el capital comunitario esta la fortaleza, confianza y oportunidad para la revitalización de una economía que satisfaga las necesidades básicas de salud con calidad, educación con calidad, vivienda de 
calidad y recreación adecuada, sin poner en riesgo los recursos naturales, territorio e identidad.

"En nuestras comunidades hay condiciones para vivir bien"

Joven universitario de Laguna de Perlas, 2009

Hay que terminar de una vez por todas con la lógica beneficiario/benefactor y responsabilizar a los actores, es decir que las organizaciones comunitarias: jóvenes, mujeres, cooperativas, consejos de ancianos, síndicos, profesionales y líderes de las comunidades asuman su propio desarrollo. Hay que fomentar los intercambios y alianzas interinstitucionales que contribuyan al empoderamiento comunitario y organización.

\section{Sinergia: elemento clave para el desarrollo con identidad}

Si partimos de que el concepto de "Sinergia" en términos organizacionales, hace referencia a la coherencia y complementariedad que debe haber en cada una de las organizaciones locales, líderes comunitarios, instituciones del Estado, proyectos con presencia en las comunidades, las iglesias y organismos no gubernamentales que desarrollan procesos encaminados al fortalecimiento del poder local y desarrollo con identidad de las comunidades.

Es decir, que tiene que ver con la cualidad de todos los actores con presencia en las comunidades de trabajar por el bien común en donde el todo es más que la sumatoria del trabajo de cada uno de los actores. En otras palabras el resultado del trabajo en consenso y compartido es superior al que se hubiera producido de manera aislada.

Cuando esto sucede es que se puede hablar de la existencia de la sinergia comunitaria e institucional. En donde todos los actores con presencia en las comunidades, trabajan orientados y guiados por una misma visión y misión de desarrollo comunitario. La estructura social de la comunidad está constituida por elementos que se relacionan entre sí, de tal manera que si uno de ellos cambia o sufre modificaciones, los otros elementos también cambian. Estos elementos son: la familia, las organizaciones comunitarias y las redes sociales (Nuevo FISE, 2009).

Si bien es cierto que en las comunidades de Laguna de Perlas, Awas y Haulover se tienen planes de desarrollo comunitario orientados por una visión y misión compartida y se ha tenido el acompañamiento por organismos no gubernamentales como Acción Medica Cristiana, FADCANIC y las universidades BICU y URACCAN principalmente; según los comunitarios, no se ha logrado que todos los líderes, proyectos, organizaciones gubernamentales y no gubernamentales e instituciones del Estado participen y asuman con responsabilidad social el desarrollo comunitario e institucional de las comunidades. 
"Algunos líderes no hacen bien su trabajo (...) una vez que salen electos de van para Bluefields"

Comunitario de Laguna de Perlas, 2009

Es decir, que no se ha logrado articular esfuerzos y optimizar los recursos para el aseguramiento de mejores condiciones de vida de las familias en las comunidades -maximizando resultados-.

“...aquí cada quien trabaja por su cuenta, es decir, que ya no hay unidad...ahora todo se relaciona con los partidos políticos de Managua..." (Líder de la comunidad de Awas)

En las entrevistas realizadas a personas generadoras de opinión y líderes de las comunidades coinciden en la necesidad de aprovechar los diferentes espacios de participación para la construcción y articulación de planes de desarrollo comunitario. En el Capítulo IV de la Ley de Municipio se refiere específicamente a los mecanismos, formas y expresiones que existen para que los Gobiernos Municipales puedan promover la participación de la ciudadanía en la gestión y ejecución de las diferentes obras de desarrollo local impulsado por las alcaldías.

Entre los espacios mencionados, recobran importancia los Consejos Municipales, las Asambleas Comunitarias y Territoriales, Los Consejos de Ancianos y la existencia misma de los Gabinetes del Poder Ciudadana, particularmente en el caso de Laguna de Perlas.

De acuerdo a los participantes de uno de los grupos focales, esto podría ayudar a superar las contradicciones y conflictos que muchas veces han surgido entre las instancias municipales, las comunitarias y la presencia de otras instancias del estado y ONGs, al momento de poner en marcha programas y proyectos encaminados mejorar la realidad social, económica, cultural y ambiental de las comunidades.

"Aquí en su mayoría solo la organización han apoyado a la comunidad"

Líder de la comunidad de Halouver, 2009

Lo anterior evidencia de forma clara la necesidad de construir una sinergia comunitaria, entendida como la acción y creación de espacios en donde los diferentes actores aporten desde sus organizaciones e instituciones buena en un marco de respeto mutuo, en donde las relaciones sean horizontales. Es decir, que los diferentes actores se unan con espíritu innovador, creativo y proactivo compartiendo principios, objetivos, visión y misión del desarrollo soñado por las comunidades y por lo que hay que trabajar en equipo y acompañar a las comunidades para que juntos alcancen su autodesarrollo. 
Algunas de las personas de las comunidades que fueron entrevistadas consideran que el Estado debe de ser el principal facilitador de procesos para la construcción de sinergia institucional y comunitaria. Para asegurar la movilización y participación directa de los ciudadanos y ciudadanas en las tomas de decisiones alrededor de su propio desarrollo, por supuesto todo en el marco de las leyes, particularmente las leyes No. 28 y 445 .

"Solamente si recuperamos nuestras costumbres, nuestros valores ancestrales... vamos a poder salir adelante"

Líder de la comunidad de Awas, 2009.

Líderes, lideresas de las comunidades consideran que revitalizar las buenas prácticas ancestrales podría significar un aporte importante para la construcción de sinergia en las comunidades y vencer las desigualdades y exclusión social existente en las comunidades. También se coincide en que la diversidad existente en cada una de las comunidades debe de verse como fuente de oportunidad y fortaleza para la innovación y construcción de consenso en donde la principal ganadora sea la comunidad, es decir todos por el bien común, -un desarrollo con identidad-.

Tomando en cuenta lo anterior se puede afirmar que solamente a través de la revitalización del tejido de relaciones e interacciones comunitarias ancestrales es posible la construcción de una sinergia interinstitucional y comunitaria.

\section{Conclusiones}

Las comunidades cuentan con diversidad étnica y sociocultural. De igual manera autodefinen institucionalidad como sus propias reglas, principios y valores ancestrales e históricos.

La institucionalidad y la organización social de las comunidades se vinculan por aspectos sociales, familiares, actividades económicas, culturales, territorialidad y comunidad.

La familia en las comunidades constituye el principal grupo de pertenencia y referencia social.

En las comunidades se establecen y reconocen como elementos de identidad sus conocimientos ancestrales, historia y tradiciones, sistema de valores, creencias, formas de organización tierra, territorio y recursos naturales. Se reconoce la existencia del marco jurídico autonómico, que respalda el ejercicio y acciones de las organizaciones comunitarias y liderazgo natural. 
También se considera que esta institucionalidad propia de las comunidades se ve obstaculizada y en cierta medida acaparada por un nuevo concepto de gobierno; alcaldía municipal (gobierno municipal).

Se percibe a lo interno de las comunidades la crisis de liderazgo comunitario, lo que provoca una preocupación por la perdida en el uso y manejo de los recursos naturales.

Por el trabajo y las acciones desarrolladas por FADCANIC, URACCAN, AMC, se les consideran como una buena práctica.

Se reconoce que existe la necesidad de construir una sinergia comunitaria, y para esto el Gobierno Regional debe de ser el principal facilitador.

El desarrollo con identidad de las comunidades esta cimentado en el fortalecimiento de la institucionalidad sociocultural y en la reconstrucción del tejido social, del uso adecuado y sostenible del territorio y los recursos naturales.

\section{Lista de Referencias}

Bebbington, A. (2005). Estrategias de vida y estrategias de intervención: el capital social y los programas de superación de la pobreza del libro Aprender de la experiencia El capital social en la superación de la pobreza de la Editora Irma Arriagada; Chile.

Dagmar R. Serrano, C. (2005). Programas de superación de la pobreza y el capital social. Evidencias y aprendizajes de la experiencia en Chile. Chile.

Deruyttere, A. (2001). Pueblos indígenas, globalización y desarrollo con identidad: algunas reflexiones de estrategia. Editorial BID.

FADCANIC. (2009). Informe de Gestión 2008-2009. Recuperado el 20 de Noviembre del 2009, de www.fadcanic.org.ni .

Gutiérrez, N. (2006). Cosmovisión y uso cultural de los recursos de recursos naturales del pueblo indígena de Ulwa de la comunidad de Karawala. Tesis, URACCAN.

Masson, C. (2005). ¿Es posible fortalecer la institucionalidad en el Perú? Recuperado el 15 de Julio 2007, en www.monografias.com

PNUD, (2005). Informe de Desarrollo Humano de la Costa Caribe. IDHCC-Managua, Nicaragua 
Ulloa, L., F. et al. (2008). Propuesta Pedagógica para el Desarrollo. FUNARTE, Estelí Nicaragua.

URACCAN- CEIMM (2004). Capital Social de las Regiones Autónomas. Editora URACCAN, Managua Nicaragua.

Villada, J. G. (2002). Institucionalidad y Desarrollo. Editorial BID.

Zapata, Y, Gurdian, G., Ruiz, P. (2006). Informe sobre Participar, Deliberar y Concertar para Construir la Ciudadanía Multiétnica e Intercultural en la Sociedad y Estado Nicaragüense. Consejo Nacional de Planificación Económica y Social. CONPES.

\section{Comunicación Personal}

MSc. Socorro Wood, Pobladora de Laguna de Perlas.

MSc. Nubia Ordoñez, URACCAN, pobladora de Laguna de Perlas.

Lic. Kart Thinkam, Laguna de Perlas.

Dr. Samuel Amador, AMC.

Norman Howard, PNUD-Originario de Laguna de Perlas.

Wesly Williams, Poblador de Laguna de Perlas.

Ingrid Cuthber, Pobladora de Laguna de Perlas.

Roberto Cuthber, Alcalde de Laguna de Perlas. 